\title{
Modelo de cálculo para o cisalhamento do concreto nos conectores Crestbond
}

\author{
Hermano de Sousa Cardoso ${ }^{1 *}$, Rodrigo Barreto Caldas ${ }^{1}$, Ricardo Hallal Fakury ${ }^{1}$, \\ Gustavo de Souza Veríssimo² e Ricardo Laguardia Justen de Almeida ${ }^{2}$ \\ ${ }^{1}$ Universidade Federal de Minas Gerais, Programa de Pós-Graduação em \\ Engenharia de Estruturas, Av. Antônio Carlos, 6627 - Escola de Engenharia, \\ Bloco I - 40 andar - Sala 4215- Pampulha - Belo Horizonte - MG - Brasil, \\ hermanocardoso@hotmail.com, rbcaldas@gmail.com, fakury@dees.ufmg.br \\ 2 Departamento de Engenharia Civil, Universidade Federal de Viçosa, Av. P. H. \\ Rolfs, s/n, Campus Universitário, Viçosa, MG, Brasil, \\ gustavo@ufv.br, ricardoljalmeida@gmail.com
}

\section{Design model for the concrete shearing in the Crestbond shear connectors}

\begin{abstract}
Resumo
Este artigo propõe um modelo semiempírico para estimar a resistência de conectores Crestbond (CR) contínuos e descontínuos, quando o cisalhamento do concreto governa o colapso da conexão. Esse modelo segue a tendência de padronizar as expressões para o dimensionamento de conectores constituídos por chapas de aço com recortes regulares, conhecidos no âmbito internacional como composite dowels, visto que esses dispositivos apresentam comportamentos semelhantes. A capacidade do concreto que preenche a abertura do conector em resistir ao cisalhamento é estimada, sob a perspectiva do uso de diferentes geometrias do conector, considerando fatores de ajuste nas equações já conhecidas e atualmente em uso. Para tal, o estudo teórico foi conduzido a partir de análises de ensaios de cisalhamento reduzidos por meio de simulações via elementos finitos. Por fim, o modelo proposto foi comparado com resultados de ensaios disponíveis na literatura, mostrando-se eficaz.
\end{abstract}

Palavras-chave: Crestbond, ensaios de cisalhamento, composite dowels, conectores de cisalhamento, vigas mistas

\begin{abstract}
This paper proposes a semi-empirical model to estimate the strength of continuous and discontinuous Crestbond shear connectors (CR), whenever the concrete shearing is the predominant failure mode of the connection. This model follows the tendency to standardize the design expressions for connectors composed by steel plate with regular cutouts, which are known internationally as composite dowels, since these devices behave similarly. The shear capacity of the concrete which fills the recesses of any type of composite dowel geometry is estimated considering different adjustments factors used in the equations already known and commonly employed. For this propose, the theoretical study was carried out from the analyzes of single push-tests through the finite element simulation. Finally, the proposed model was compared with tests outputs reported in the literature, showing good agreement among them.
\end{abstract}

Keywords: Crestbond, push-tests, composite dowels, shear connectors, composite beams

* autor correspondente 


\section{Introdução}

Os conectores de cisalhamento constituídos por chapas de aço com recortes regulares, conhecidos internacionalmente como composite dowels, constituem um meio de conexão eficiente entre o aço e o concreto nos elementos estruturais mistos. Esses dispositivos têm sido empregados em vários países europeus em vigas de pontes e viadutos. Esses conectores podem possuir diversas geometrias, sendo muito empregadas as denominadas clothoidal-shaped (Figura 1a) e puzzle-shaped (Figura 1b), cujas expressões para dimensionamento podem ser encontradas nos guias de projeto PreCo-Beam (Seidl et al., 2013a) e PreCo+ (Seidl et al., 2013b), respectivamente. Logo após a publicação desses guias, as prescrições de projeto e as expressões para dimensionamento dos dois conectores foram incluídas na aprovação técnica alemã Z26.4-56 (2013). Também de larga utilização na Europa é o conector chamado de Perfobond (Figura 1c) que, no lugar dos recortes, apresenta aberturas circulares regularmente espaçadas. O Perfobond tem um modelo analítico de dimensionamento proposto por Oguejiofort e Hosain (1997).

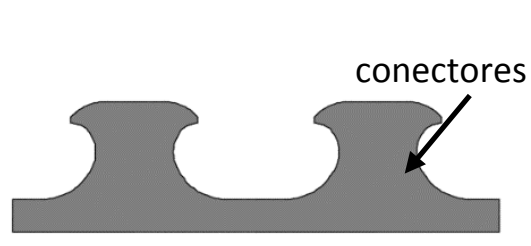

(a) Clothoidal-shaped

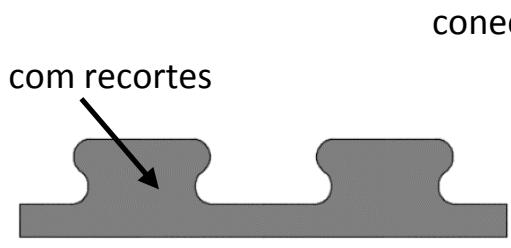

(b) Puzzle -shaped conector com aberturas

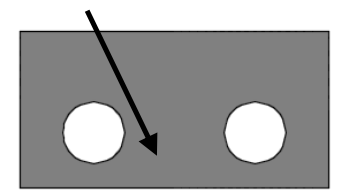

(c) Perfobond - geometria utilizada no estudo de Oguejiofor e Hosain (1994)

Figura 1 - Conectores constituídos por chapas

Outro conector constituído por chapa com recortes regulares, denominado Crestbond (CR), tem sua forma e parâmetros geométricos mostrados na Figura 2. Esses conectores possuem "dentes" de aço mais altos e menos largos em relação aos com geometria puzzle-shaped. A concepção da geometria do conector Crestbond foi desenvolvido por Veríssimo (2007) no Brasil, como uma alternativa aos conectores pino com cabeça, os mais utilizados no país. Em Cardoso et al. (2018) são fornecidos o estado da arte e um estudo numérico envolvendo os conectores CR em ensaios de cisalhamento padrão (standard push test). 


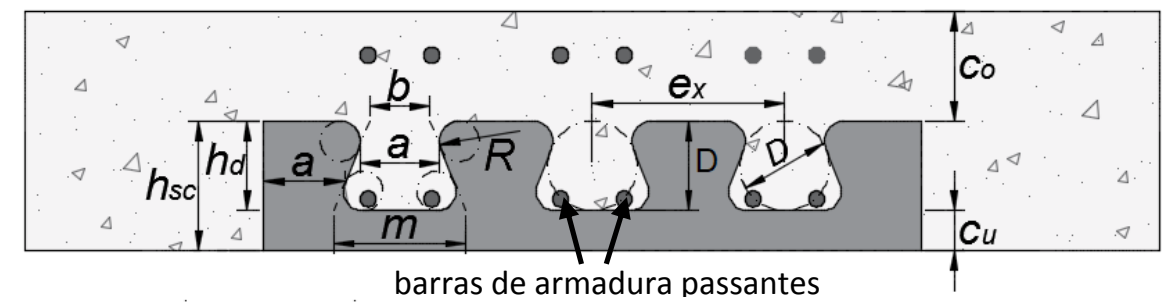

$$
\begin{aligned}
D= & \text { diâmetro do círculo inscrito no } \\
& \text { dowel de concreto } \\
R= & \text { raio de curvatura } \\
h_{d}= & \text { altura do dowel de aço }=D
\end{aligned}
$$

$$
\begin{aligned}
& c_{o}=\text { cobrimento superior de concreto } \\
& c_{u}=\text { cobrimento inferior de concreto } \\
& e_{x}=\text { passo do conector }=2,155 D \\
& m=1,486 D ; b=0,450 \mathrm{~m} ; a=2,876 R+0,250 D
\end{aligned}
$$

Figura 2 - Forma e parâmetros geométricos do conector Crestbond (CR).

Os conectores Perfobond, por possuírem aberturas que não atingem a face superior (geometria fechada), têm um comportamento mais rígido e menos dúctil que os conectores com recortes regulares (possuem grandes espaços abertos na face superior - geometria aberta). Além disso, esses conectores possuem sempre como estado-limite último a ruptura por cisalhamento do concreto. Por outro lado, nos conectores de geometria aberta, precisamente por causa da menor rigidez e maior ductilidade, podem prevalecer outros estados-limites últimos. Além do cisalhamento do concreto, podem ocorrer o pry-out, fenômeno abordado com maiores detalhes nos trabalhos de Feldmann et al. (2016) e Classen e Hegger (2017), e o colapso por flexão dos "dentes" de aço do próprio conector. Cobrimento de concreto reduzido favorece a ocorrência do pry-out e chapas mais finas e aberturas maiores do conector favorecem a ocorrência da flexão dos "dentes". No entanto, na maioria das situações práticas, as dimensões desses parâmetros são tais que prevalece o cisalhamento do concreto como modo de colapso. O primeiro modelo analítico para o dimensionamento dos conectores Crestbond quanto à ruptura por cisalhamento do concreto foi proposto por Veríssimo (2007), sendo seguido pelos modelos de Silva (2011) e Dutra (2014). Todos esses três modelos apresentam bons resultados, sendo que os dois primeiros foram desenvolvidos a partir de uma concepção analítica mais antiga apresentada por Oguejiofort e Hosain (1997) especificamente para o conector Perfobond (Figura 1c).

Este trabalho pode ser considerado como uma continuidade ao de Cardoso et al. (2018) e apresenta o desenvolvimento de um novo modelo analítico para o cálculo da capacidade resistente de conectores Crestbond quando a ruptura por cisalhamento do concreto governa o colapso da conexão. Para isso, foram considerados dados de modelos experimentais e numéricos disponíveis na literatura, além dos resultados da 
simulação numérica de Cardoso et al. (2018), nos quais foi observada a ocorrência desse estado-limite último.

\section{Cisalhamento dos Componentes de Concreto}

Na Figura 3 é mostrada a transferência de forças entre o perfil de aço e o concreto quando são utilizados conectores formados por chapas com recortes regulares (composite dowels). Para limitar o deslizamento relativo entre ambos, a região inferior do conector fica sujeita a uma força oriunda de esforços de cisalhamento $\left(F_{v}\right)$. Desse modo, outra força de mesma magnitude e de sentido contrário atua no conector, gerando o equilíbrio. Essa força faz com que surjam tensões de contato na interface entre os componentes (ou dowels) de aço ("dente" de aço) e de concreto (delimitado pela região entre os componentes de aço) da conexão, que interagem entre si.

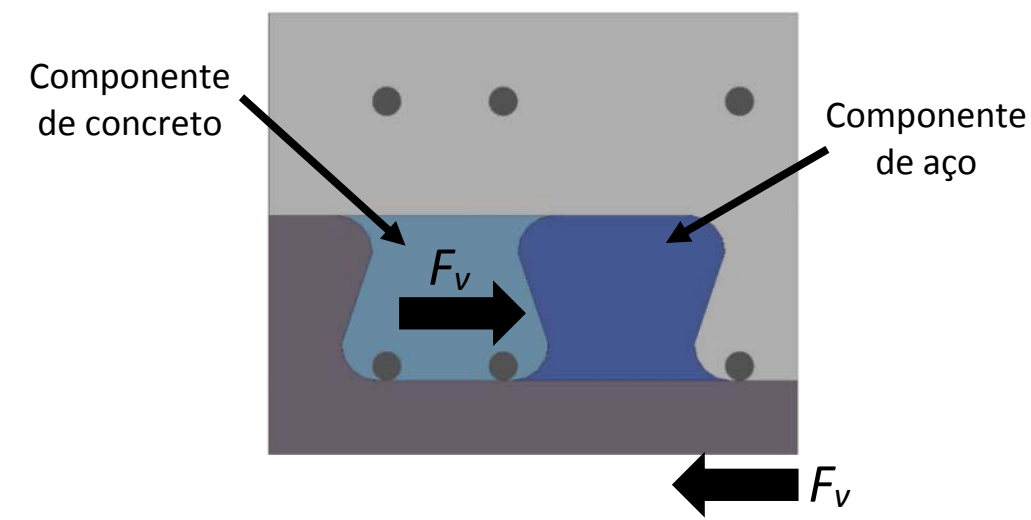

Figura 3 - Introdução de esforços de cisalhamento em conectores formados por chapas.

Segundo a aprovação técnica Z-26.4-56 (2013), a capacidade de um componente de concreto dos conectores clothoidal-shaped e puzzle-shaped em resistir esforços de cisalhamento pode ser estimada pela seguinte equação:

$q_{u, s h}=\eta_{D} e_{x}^{2} \sqrt{f_{c}}\left(1+\rho_{D}\right)$

com

$\rho_{D}=\frac{E_{r} A_{b}}{E_{c m} A_{D}}$

onde $e_{x}$ é o passo do conector (ver Figura 2), $f_{c}$ é a resistência à compressão do concreto, $E_{r}$ e $A_{b}$ são o módulo de elasticidade e a área total das barras da armadura passante, respectivamente, $E_{c m}$ é o módulo de elasticidade secante do concreto e $A_{D}$ é a área do componente de concreto, definida como a área compreendida entre dois componentes de aço. Ainda na Equação 1, $\eta_{D}$ indica o fator de geometria do conector, cujo valor, em 
projetos de pontes mistas, pode ser tomado como igual a $\eta_{D, C L}=3-\left(e_{x} / 180\right)$ para conectores clothoidal-shaped e a $\eta_{D, P S}=2-\left(e_{x} / 400\right)$ para conectores puzzle-shaped (Seidl et al., 2013a e 2013b; Z-26.4-56, 2013).

\section{Cisalhamento dos Componentes de Concreto}

\subsection{Considerações Iniciais}

Alguns procedimentos de modelagem utilizados em Cardoso et al. (2018) -como a malha de elementos finitos, leis constitutivas dos materiais e método de convergência - foram mais uma vez utilizados neste artigo na simulação de modelos reduzidos para as análises individuais dos componentes de concreto. Para tal, foi utilizado o software comercial de elementos finitos Abaqus 6.14 (Hibitt et al., 2014). As geometrias de conectores estudadas e comparadas neste item foram as dos conectores puzzle-shaped e Crestbond CR56b ( $D=56 \mathrm{~mm}, e_{x}=121 \mathrm{~mm}, c_{o}=69 \mathrm{~mm}, c_{u}=25 \mathrm{~mm}-$ ver Figura 2).

No início do Subitem 3.2, é realizada a simulação de uma primeira bateria de modelos com conectores puzzle-shaped. O conector puzzle-shaped além de apresentar uma geometria similar ao Crestbond, tem comportamento conhecido, sendo descrito em diversos estudos, na aprovação técnica Z-26.4-56 (2013) e em guias de dimensionamento (Seidl et al, 2013a e b). Assim, ao se comparar os resultados numéricos e analíticos dos modelos com conector puzzle-shaped, verificou-se que os procedimentos numéricos aferidos em Cardoso et al. (2018) continuavam válidos e foram adotados para o estudo de caso do presente trabalho.

Desse modo, foram realizadas novas simulações com conectores Crestbond, possibilitando que, ao final, se estimasse o fator de ajuste $\eta_{D}$ para esses conectores. Esse procedimento possibilitaria o emprego da Equação 1 para fins de projeto e dimensionamento dos conectores Crestbond. Para tal, foram determinados valores de $\eta_{D}$ em duas situações distintas, a de alto confinamento (ainda no Subitem 3.2) e a de baixo confinamento (Subitem 3.3). As situações de alto confinamento são aquelas em que se utilizam alta taxa de armadura no entorno dos conectores, usualmente encontradas em vigas mistas de pontes, nas quais são considerados os fatores de ajustes presentes na aprovação técnica Z-26.4-56 (2013). Ao contrário, as situações de baixo confinamento são observadas, na maioria dos casos, nas vigas mistas de edifícios. 


\subsection{Estudo Numérico-teórico do Cisalhamento dos Componentes de Concreto em Situações de Alto Confinamento.}

Para que ocorra o cisalhamento do componente de concreto, sem que haja o desprendimento da laje de concreto em relação ao perfil de aço, a base da laje foi restringida segundo as direções $x, y$ e $z$ (Figura 4). Essa restrição, nos três eixos, tem como objetivo simular a forma com que esses conectores são utilizados em pontes, com continuidade da laje de concreto e do conector, e com alta taxa de armadura - situação de alto confinamento. As geometrias dos modelos e as condições de contorno foram baseadas no ensaio de cisalhamento adaptado para a análise de um componente de concreto realizada no estudo numérico-experimental de Classen e Gallwoszus (2016).

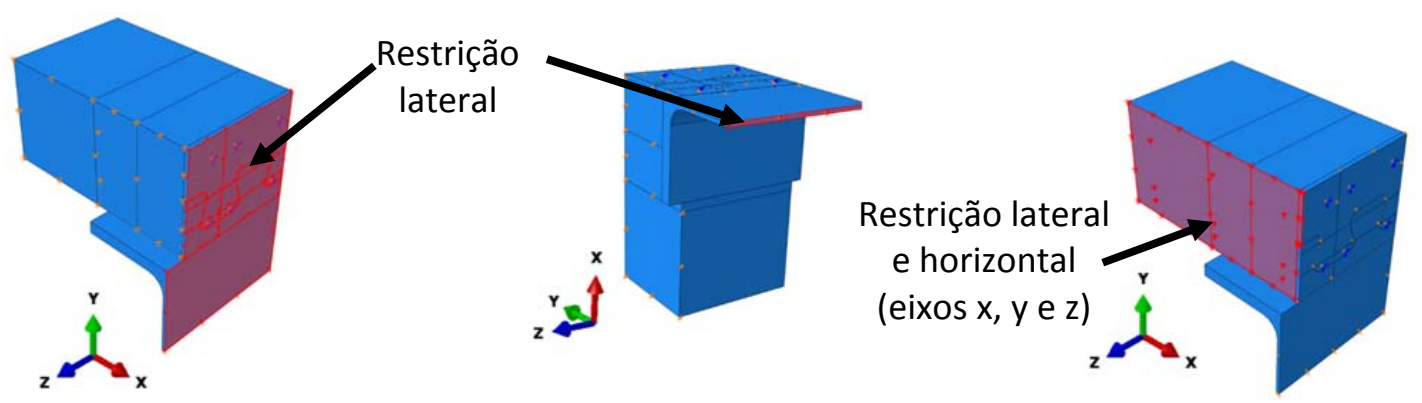

Figura 4 - Condições de contorno nos modelos reduzidos para a análise dos componentes de concreto.

Nas simulações do conector puzzle-shaped realizadas neste trabalho foram adotados os seguintes parâmetros: passo $\left(e_{x}\right)$ igual a $150 \mathrm{~mm}$; duas barras de armadura passando no componente de concreto com $10 \mathrm{~mm}$ de diâmetro; conector com espessura $\left(t_{s c}\right)$ igual a $12 \mathrm{~mm}$; e, a resistência à compressão do concreto $\left(f_{c}\right)$ variando entre $20 \mathrm{MPa}$ e $50 \mathrm{MPa}$. Na Tabela 1 é apresentado um resumo com as principais propriedades dos modelos analisados, bem como as forças analíticas $\left(P_{u, T e o}\right)$ e máximas numéricas $\left(P_{u, N u m}\right)$. O arranjo de armaduras utilizado nos ensaios apresentados por Veríssimo (2007) é semelhante ao que usualmente é utilizado em vigas mistas de edifício (baixo confinamento), não sendo indicado, portanto, para o estudo de caso do presente subitem (alto confinamento). Com isso, a disposição das barras de armadura utilizadas na modelagem foi baseada no trabalho experimental de Přivřelová (2016) (Figura 5) que estudou conectores com geometria clothoidal-shaped com duas barras passantes entre os componentes. Segundo Seidl et al. (2013a), o uso de pelo menos duas barras passantes promove elevada capacidade resistente e alta ductilidade do conector. 
Neste estudo optou-se em utilizar as dimensões de todos componentes (conector, armadura e perfil) tão próximas quanto possíveis daquelas dos ensaios de Veríssimo (2007). Contudo, o passo dos conectores $\left(e_{x}\right)$ foi tomado como igual a $150 \mathrm{~mm}$, valor mínimo normatizado para os puzzle-shaped, que têm todas as suas dimensões em função desse passo (Figura 2) (nos ensaios de Veríssimo, 2007, ex é igual a 121 mm).

A força $P_{u, \text { Teo }}$ foi estimada utilizando a Equação 1. Na última coluna da Tabela 1 são apresentadas as razões entre as forças $P_{u, N u m}$ e $P_{u, \text { Teo, }}$ obtendo-se uma média de 0,975. Dessa forma, conclui-se que o modelo numérico é capaz de prever adequadamente o comportamento de conectores puzzle-shaped.

Tabela 1 - Propriedades de modelos com conectores puzzle-shaped e resultados obtidos.

\begin{tabular}{|c|c|c|c|c|c|c|c|c|c|c|}
\hline Modelo & $\begin{array}{c}e_{x} \\
\mathrm{~mm}\end{array}$ & $\begin{array}{c}A_{D} \\
\mathrm{~mm}^{2}\end{array}$ & $\begin{array}{c}A_{b} \\
\mathrm{~mm}^{2}\end{array}$ & $\begin{array}{c}f_{c} \\
\mathrm{MPa}\end{array}$ & $\begin{array}{c}\text { Фs } \\
\mathrm{mm}\end{array}$ & $\eta_{D}$ & $\rho_{D}$ & $\begin{array}{c}P_{u, \text { Teo }} \\
\mathrm{kN}\end{array}$ & $\begin{array}{c}P_{u, N u m} \\
\mathrm{kN}\end{array}$ & $\frac{P_{u, \mathrm{Num}}}{P_{u, \mathrm{Teo}}}$ \\
\hline PZrest-C20 & 150 & 3003,8 & 157,1 & 20,0 & 10,0 & 1,625 & 0,41 & 229,82 & 229,81 & 1,000 \\
\hline PZrest-C25 & 150 & 3003,8 & 157,1 & 25,0 & 10,0 & 1,625 & \begin{tabular}{|l|l|}
0,38 \\
\end{tabular} & 251,29 & 252,14 & 0,988 \\
\hline PZrest-C30 & 150 & 3003,8 & 157,1 & 30,0 & 10,0 & 1,625 & 0,36 & 275,36 & 272,16 & 1,011 \\
\hline PZrest-C35 & 150 & 3003,8 & 157,1 & 35,0 & 10,0 & 1,625 & 0,34 & 288,47 & 290,46 & 0,993 \\
\hline PZrest-C40 & 150 & 3003,8 & 157,1 & 40,0 & 10,0 & 1,625 & \begin{tabular}{|l|}
0,33 \\
\end{tabular} & 296,89 & 307,40 & 0,966 \\
\hline PZrest-C45 & 150 & 3003,8 & 157,1 & 45,0 & 10,0 & 1,625 & 0,32 & \begin{tabular}{|l|}
303,71 \\
\end{tabular} & 323,24 & 0,940 \\
\hline PZrest-C50 & 150 & 3003,8 & 157,1 & 50,0 & 10,0 & 1,625 & 0,31 & 311,06 & 338,17 & 0,920 \\
\hline
\end{tabular}

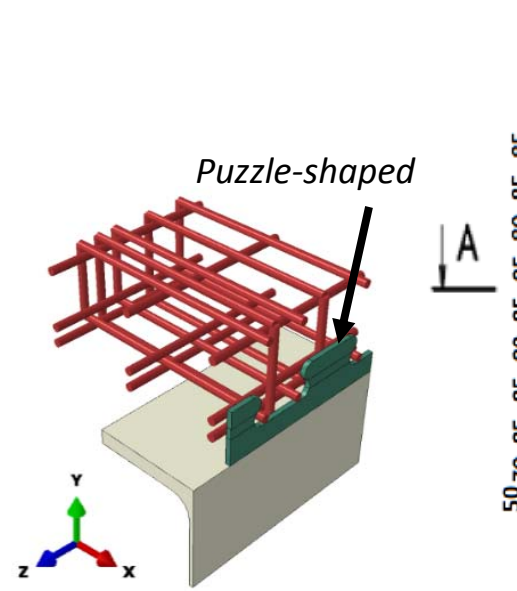

(a) Modelo numérico com a laje suprimida para ilustrar o arranjo da armadura de aço

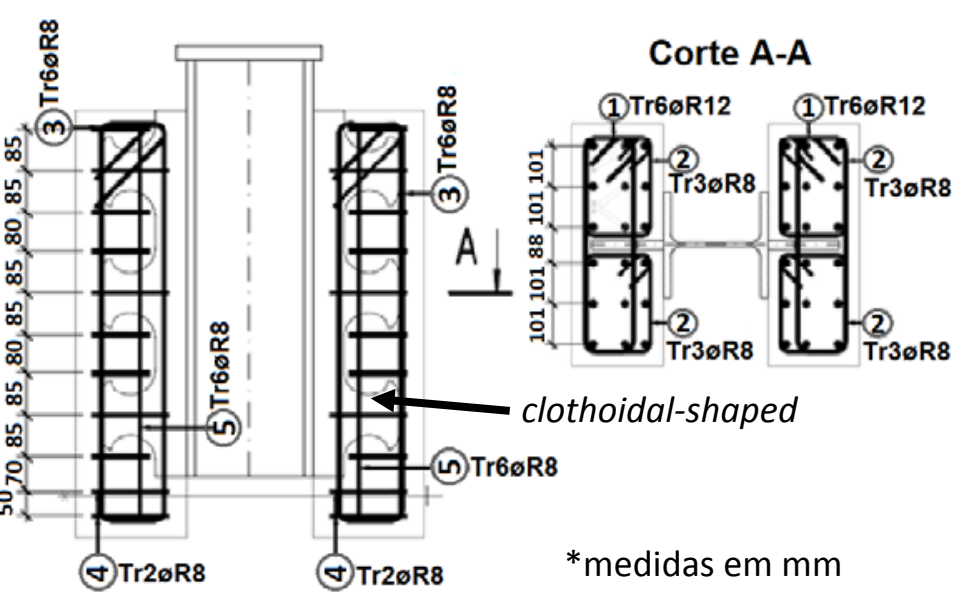

(b) Modelo experimental de Přivřelová (2016)

Figura 5 - Análise de conectores formados por chapas de aço com recortes regulares.

Seidl et al. (2013a) mencionam que quando os conectores compostos por chapas com recortes são continuamente solicitados, e o estado-limite é o cisalhamento da laje de concreto, o comportamento estrutural pode ser descrito em três etapas distintas. Essas 
etapas estão representadas nos dois diagramas de força versus deslizamento dispostos na Figuras 6, onde o primeiro contém a idealização proposta por Seidl et al. (2013a) (Figura 6a) e o segundo contendo a curva do modelo numérico PZrest-C35 e a reta representativa do modelo analítico (Figura 6b). Na primeira etapa, a interação entre os componentes de aço e concreto apresenta uma resposta linear, até ser alcançada a força $P_{a d h}$, caracterizada pelo rompimento da aderência mecânica na interface entre os componentes. No início da segunda etapa, há um trecho com rigidez nula que designa o esmagamento do concreto próximo à região da interface entre os componentes. Posteriormente, a força se eleva, dando continuidade ao processo de esmagamento e, ocorrendo também a fissuração do concreto, até o alcance da força máxima $P_{\max }$. $O$ dano do concreto à compressão no início da segunda etapa e no incremento correspondente à força $P_{\text {max }}$, podem ser observados nas Figuras $7 \mathrm{a}$ e $7 \mathrm{~b}$ adiante, respectivamente. $\mathrm{Na}$ terceira etapa, é evidenciado o comportamento pós-crítico, sendo que o decréscimo de rigidez no descarregamento depende das características mecânicas dos aços do conector e das barras da armadura e do concreto.

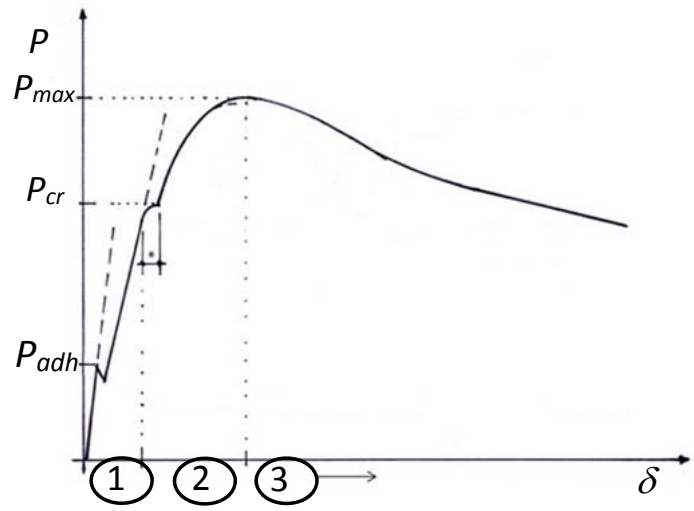

(a) Diagrama força versus deslizamento relativo sugerido para o cisalhamento do concreto (Adaptado de Seidl et al., 2013a)

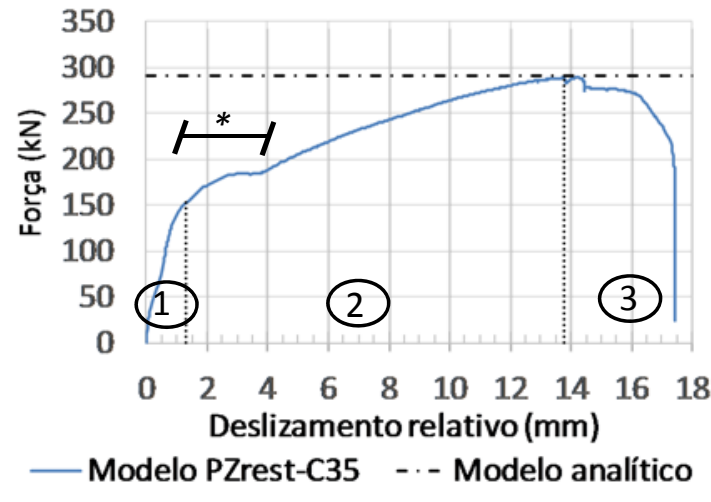

(b) Diagrama força versus deslizamento relativo do modelo numérico PZrest-35 e reta representativa do modelo analítico

Figura 6 - Falha por cisalhamento dos componentes de concreto.

Visto que o modelo numérico com conectores puzzle-shaped levou a resultados com boa concordância com o modelo analítico, realizaram-se novas simulações de modelos reduzidos com conectores Crestbond CR56b (Figura 8a). Nessas simulações, com exceção do valor de $e_{x}$, mantiveram-se as características dos modelos com conectores puzzle-shaped, como as propriedades mecânicas dos materiais e a espessura do conector. $\mathrm{O}$ valor adotado para $e_{x}$ foi o mesmo dos protótipos ensaiados por Veríssimo (2007), de $121 \mathrm{~mm}$ (Figura 8). A armadura utilizada nos modelos numéricos reduzidos 
com conectores Crestbond também foi baseada no arranjo do estudo experimental de Přivřelová (2016) (Figuras 5b e 9a).
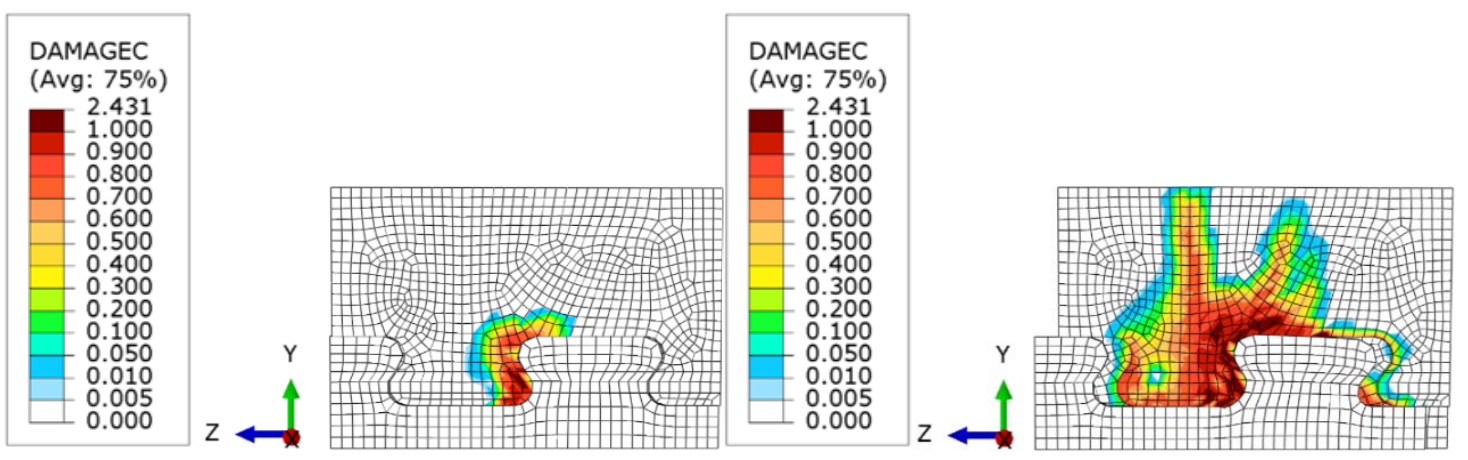

(a) Dano inicial próximo a interface entre os componentes de aço e concreto

(b) Dano no incremento correspondente à força máxima

Figura 7 - Representação do dano no concreto por compressão no modelo PZrest-C35

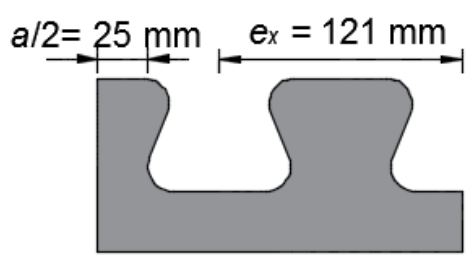

(a) Modelo reduzido para análise dos componentes de concreto

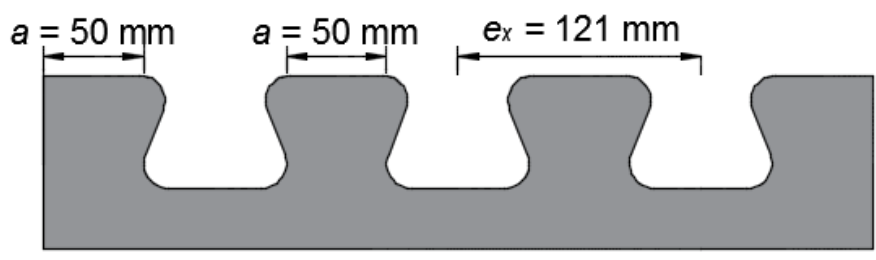

(b) Modelos dos ensaios de cisalhamento

Figura 8 - Geometrias de conectores Crestbond CR 56b utilizadas na modelagem numérica.

Na Tabela 2 é apresentado um resumo contendo as propriedades dos modelos com Crestbond, variando-se apenas a resistência à compressão do concreto - entre $20 \mathrm{MPa}$ a $50 \mathrm{MPa}$. Nas três últimas colunas dessa tabela são mostrados os resultados finais das análises contendo as forças $P_{u, N u m}$ e $P_{u, \text { Teo }}$ e a razão entre elas. Os valores de $P_{u, t e o}$ foram determinados de acordo com a Equação 1, em que o valor adotado para o parâmetro $\eta_{D}$ foi de 2,204. 0 valor de $\eta_{D}$ foi obtido por meio de regressão analítica, de modo que a razão média entre as forças máximas numérica e analítica fosse igual a 1,0. Na Figura 9b, são comparadas a curva numérica do modelo CrestRest-C35 e a reta analítica que define a capacidade estimada do modelo (ver caraterísticas do modelo na Tabela 2).

O valor de $\eta_{D}$ igual a 2,204, obtido para o conector Crestbond com $e_{x}$ de $121 \mathrm{~mm}$, é mais elevado se comparado com o valor de 1,625, utilizado para os conectores puzzle-shaped com $e_{x}$ de $150 \mathrm{~mm}$. Se fosse utilizado, hipoteticamente, um valor de $e_{x}$ igual a $121 \mathrm{~mm}$ para o conector puzzle-shaped, desconsiderando que seu valor normativo mínimo é igual a $150 \mathrm{~mm}$, o valor de $\eta_{D}$ para esse conector seria de 1,697 $\left(\eta_{D, P Z}=2-e_{X} / 400\right)$. Percebe-se que esse último valor de $\eta_{D}$ para o conector puzzle-shaped ainda é 
consideravelmente menor ao valor de $\eta_{D}$ igual 2,204 obtido para o Crestbond com $e_{x}$ de $121 \mathrm{~mm}$.

Tabela 2 - Propriedades dos modelos com conectores Crestbond e resultados obtidos.

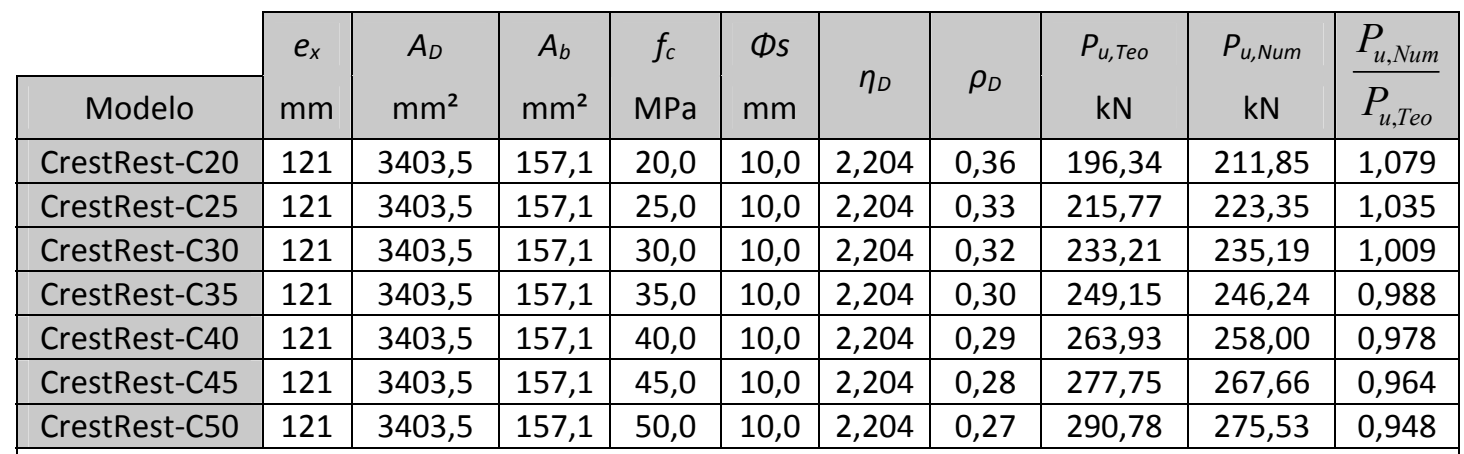

Caraterísticas em comum em todos os modelos: $t_{s c}=12,0 \mathrm{~mm}$ e $E_{r}=21.000 \mathrm{MPa} ; E_{c m}=22\left[\left(f_{c}\right) / 10\right]^{0,3}(\mathrm{EN} 1992-1-1: 2004)$

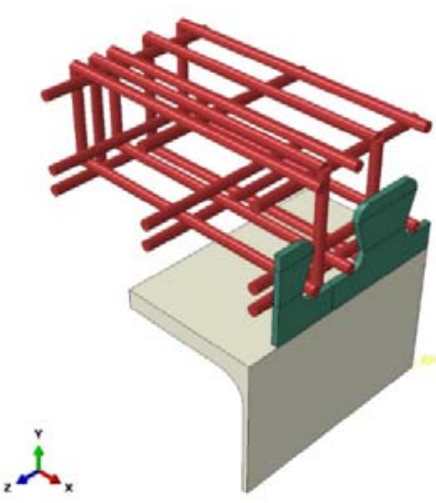

(a) Modelo numérico com a laje suprimida para ilustrar o arranjo das armaduras

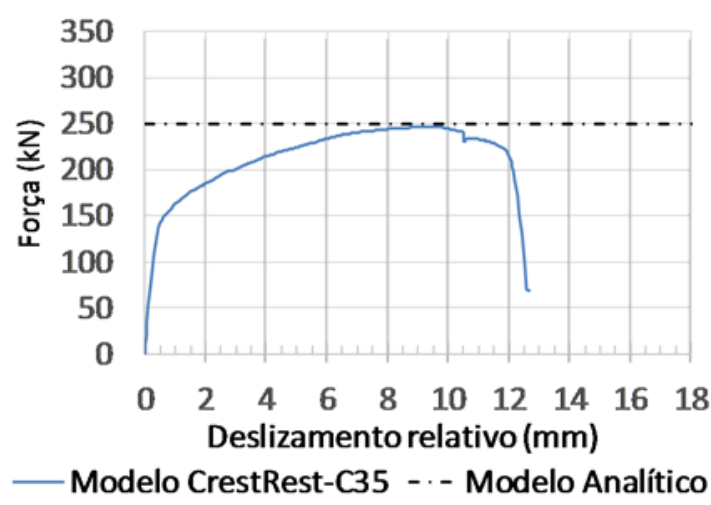

(b) Diagrama força versus deslizamento relativo do modelo CrestRest-C35.

Figura 9 - Simulação numérica de modelos simplificados para a análise dos componentes de concreto dos conectores Crestbond.

Seidl et al. (2013a) afirmam que a capacidade do conector, para o estado-limite último de ruptura por cisalhamento do concreto, é fortemente influenciada pela sua geometria. Ao observar a geometria do conector Crestbond, percebe-se que ele é formado por componentes mais altos do que os do puzzle-shaped, permitindo uma superfície de contato maior entre o aço e o concreto. Segundo Seidl et al. (2013a), elevadas tensões de contato ocorrem na interface entre os componentes de aço e de concreto. Essas tensões acompanham o formato arqueado do conector, conforme pode ser observado na Figura 10a, na qual são ilustradas as tensões de contato na interface aço-concreto em um conector puzzle-shaped. Na Figura 10b é apresentada a variável CPREES (valores em $\mathrm{MPa}$ ), na superfície do componente de aço, no incremento de força máxima para o modelo numérico CrestRest-C35, o qual foi simulado neste trabalho. Essa variável 
quantifica as tensões de contato que atuam numa superfície de uma dada instância. Como pode ser observado na Figura 10b, a distribuição das tensões de contato atuando ao longo da superfície do componente de aço do conector Crestbond se dá de forma semelhante à idealização dessas tensões para o conector puzzle-shaped (Figura 10a). Considerando que os conectores Crestbond apresentam altura $\left(h_{d}\right)$ maior que os conectores puzzle-shaped - sendo essas alturas, $h_{d, \text { Crest }}=0,464 e_{x, \text { Cret }}=56 \mathrm{~mm} \mathrm{e} h_{d, P z}=$ $0,275 e_{x, P Z}=41,25 \mathrm{~mm}$, respectivamente - acredita-se que o fator $\eta_{D}$ poderia se elevar, devido a uma maior superfície de contato entre os componentes de aço e de concreto e também a uma maior área de cisalhamento do concreto.

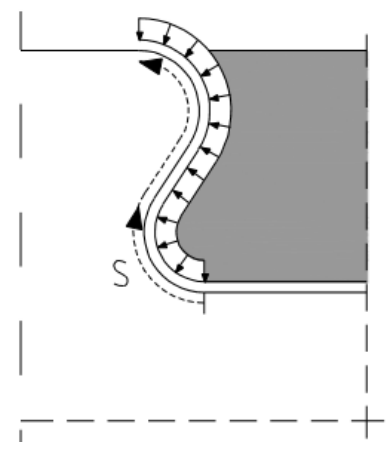

(a) Idealização das tensões de contato em conectores Puzzle - shaped (Seidl et al., 2013a)
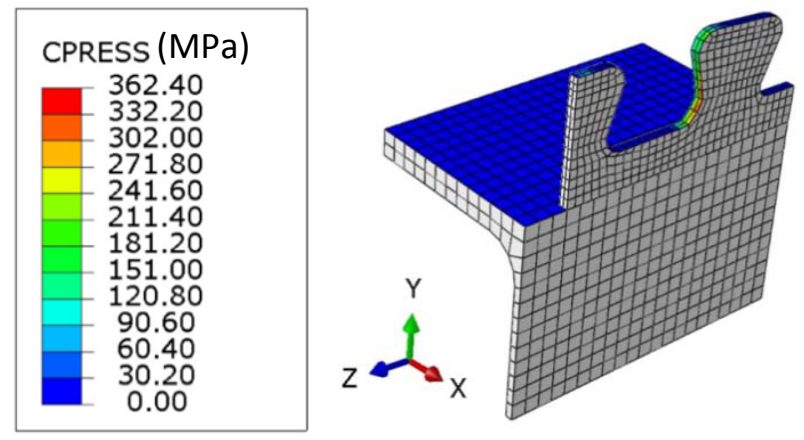

Figura 10 - Tensões de contato nos conectores de cisalhamento.

\subsection{Estudo Numérico-teórico do Cisalhamento dos Componentes de Concreto em Situações de Baixo Confinamento}

Neste item, foram realizadas simulações de modelos reduzidos com conectores Crestbond para verificar a resistência de apenas um componente de concreto em um ensaio de cisalhamento padrão. Dessa forma, ao invés de se restringir na base da laje de concreto a translação nos três eixos cartesianos, como no item anterior, restringiu-se somente a translação na direção vertical.

Na Tabela 3 adiante são apresentadas as principais características geométricas e mecânicas dos 21 modelos numéricos que foram simulados neste trabalho e são analisados neste subitem, que podem possuir duas $(2 \Phi)$, uma (1Ф) ou nenhuma barra de armadura passante $\left(A_{s 0}\right)$. As disposições das barras (Figura 11), para as simulações do presente subitem (situações de baixo confinamento), foram baseadas nos ensaios de 
cisalhamento realizados por Veríssimo (2007). Nesses modelos adotou-se o valor de $e_{x}$ igual a $121 \mathrm{~mm}$, mesmo valor utilizado para os modelos de Crestbond presentes no Subitem 3.2. Nas últimas colunas da Tabela 3 são apresentadas as forças máximas numéricas $P_{u, N u m}$ e analíticas $P_{u, T e o}$ e a razão entre elas. No cálculo de $P_{u, t e o}$ foi utilizada a Equação 1, porém era necessário, de antemão, que se determinasse o valor de $\eta_{D}$ apropriado para o Crestbond. Para tal, o valor desse parâmetro foi obtido por regressões analíticas de forma semelhante ao que foi feito no Subitem 3.2. Contudo, neste subitem, foi realizado um ajuste de $\eta_{D}$ para as diferentes disposições de armaduras analisadas, chegando-se a $\eta_{D, A s}$ igual a 0,873 quando se utilizam uma ou duas barras de armadura passantes e igual a 0,570 para nenhuma barra.

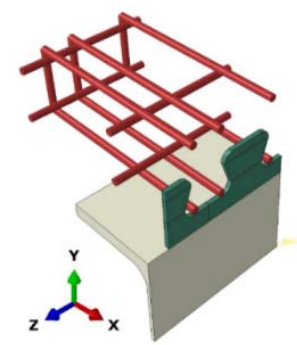

(a) Duas barras passantes

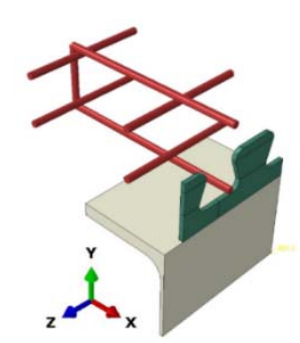

(b) Uma barra passante

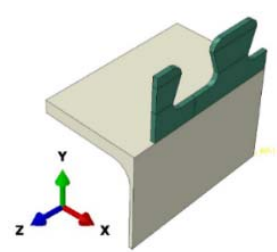

(c) Sem barra passante

Figura 11 - Disposição das armaduras em modelos numéricos para a análise do comportamento do componente de concreto de conectores Crestbond em ensaios de cisalhamento padrão.

Na Figura 12 são apresentadas as curvas numéricas de força versus deslizamento relativos dos modelos cujo $f_{c}$ é $35 \mathrm{MPa}$, para as três taxas de armadura estudadas (observar modelos na Tabela 3). Nota-se que o uso de nenhuma armadura passante diminuiu drasticamente a ductilidade do conector e a capacidade do componente de concreto em resistir esforços de cisalhamento, sendo desaconselhado.

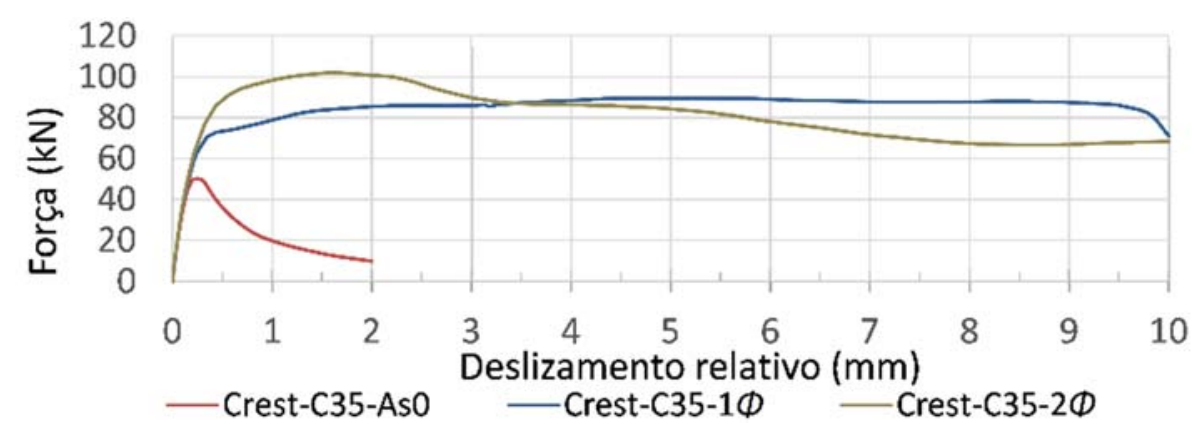

Figura 12 - Diagrama força versus deslizamento relativo dos modelos Crest-C35-As0, Crest-C35-As0-1Ф, Crest-C35-As0-2Ф. 
Tabela 3 - Propriedades dos modelos com conectores Crestbond e resultados obtidos.

\begin{tabular}{|c|c|c|c|c|c|c|c|c|c|c|}
\hline Modelo & $\begin{array}{c}e_{x} \\
\mathrm{~mm}\end{array}$ & $\begin{array}{c}A_{D} \\
\mathrm{~mm}^{2}\end{array}$ & $\begin{array}{c}A_{b} \\
\mathrm{~mm}^{2}\end{array}$ & $\begin{array}{c}f_{c} \\
\mathrm{MPa}\end{array}$ & $\begin{array}{l}\text { Фs } \\
\mathrm{mm}\end{array}$ & $\eta_{D}$ & $\rho_{D}$ & $\begin{array}{c}P_{u, T e o} \\
\text { kN }\end{array}$ & $\begin{array}{c}P_{u, \mathrm{Num}} \\
\mathrm{kN}\end{array}$ & $\frac{P_{u, \text { Num }}}{P_{u, \text { Teo }}}$ \\
\hline Crest-C20-2Ф & 121 & 3403,5 & 157,1 & 20,0 & $2 \times 10,0$ & 0,873 & 0,36 & 77,77 & 65,97 & 0,848 \\
\hline Crest-C25-2Ф & 121 & 3403,5 & 157,1 & 25,0 & $2 \times 10,0$ & 0,873 & 0,33 & 85,47 & 77,83 & 0,911 \\
\hline Crest-C30-2Ф & 121 & 3403,5 & 157,1 & 30,0 & $2 \times 10,0$ & 0,873 & 0,32 & 92,37 & 91,00 & 0,985 \\
\hline Crest-C35-2 $\Phi$ & 121 & 3403,5 & 157,1 & 35,0 & $2 \times 10,0$ & 0,873 & 0,30 & 98,69 & 10,90 & 1,033 \\
\hline Crest-C40-2Ф & 121 & 3403,5 & 157,1 & 40,0 & $2 \times 10,0$ & 0,873 & 0,29 & 104,54 & 111,74 & 1,069 \\
\hline Crest-C45-2Ф & 121 & 3403,5 & 157,1 & 45,0 & $2 \times 10,0$ & 0,873 & 0,28 & 110,02 & 120,93 & 1,099 \\
\hline Crest-C50-2Ф & 121 & 3403,5 & 157,1 & 50,0 & $2 \times 10,0$ & 0,873 & 0,27 & 115,18 & 129,29 & 1,123 \\
\hline Crest-C20-1Ф & 121 & 3403,5 & 78,5 & 20,0 & 10,0 & 0,873 & 0,18 & 67,52 & 60,84 & 0,901 \\
\hline Crest-C25-1Ф & 121 & 3403,5 & 78,5 & 25,0 & 10,0 & 0,873 & 0,17 & 74,75 & 72,96 & 0,976 \\
\hline Crest-C30-1Ф & 121 & 3403,5 & 78,5 & 30,0 & 10,0 & 0,873 & 0,16 & 81,26 & 82,29 & 1,013 \\
\hline Crest-C35-1Ф & 121 & 3403,5 & 78,5 & 35,0 & 10,0 & 0,873 & 0,15 & 87,23 & 89,55 & 1,027 \\
\hline Crest-C40-1Ф & 121 & 3403,5 & 78,5 & 40,0 & 10,0 & 0,873 & 0,15 & 92,77 & 94,58 & 1,019 \\
\hline Crest-C45-1Ф & 121 & 3403,5 & 78,5 & 45,0 & 10,0 & 0,873 & 0,14 & 97,96 & 98,41 & 1,005 \\
\hline Crest-C50-1Ф & 121 & 3403,5 & 78,5 & 50,0 & 10,0 & 0,873 & 0,14 & 102,87 & 102,62 & 0,998 \\
\hline Crest-C20-As0 & 121 & 3403,5 & 0 & 20,0 & 0 & 0,570 & 0 & 37,41 & 32,89 & 1,138 \\
\hline Crest-C25-As0 & 121 & 3403,5 & 0 & 25,0 & 0 & 0,570 & 0 & 41,83 & 39,28 & 1,065 \\
\hline Crest-C30-As0 & 121 & \begin{tabular}{|l|}
3403,5 \\
\end{tabular} & 0 & 30,0 & 0 & 0,570 & 0 & 45,82 & 44,86 & 1,022 \\
\hline Crest-C35-As0 & 121 & 3403,5 & 0 & 35,0 & 0 & 0,570 & 0 & 49,49 & 50,15 & 0,987 \\
\hline Crest-C40-As0 & 121 & 3403,5 & 0 & 40,0 & 0 & 0,570 & 0 & 52,91 & 55,41 & 0,955 \\
\hline Crest-C40-As0 & 121 & 3403,5 & 0 & 45,0 & 0 & 0,570 & 0 & 56,12 & 60,81 & 0,923 \\
\hline Crest-C50-As0 & 121 & 3403,5 & 0 & 50,0 & 0 & 0,570 & 0 & 59,16 & 64,92 & 0,911 \\
\hline
\end{tabular}

Em Cardoso et al. (2018), foram discutidos os modelos experimentais de Veríssimo (2007) com conectores Crestbond CR56b descontínuos, sendo realizada a calibração numérica desses experimentos e a análise do comportamento do conector. Contudo, nesse estudo não foi determinada quais parcelas da capacidade total seriam devidas aos componentes de concreto e à parte frontal do conector. Assim, foram simulados novamente os modelos com conectores Crestbond do trabalho de Cardoso et al. (2018), conforme se observa na Tabela 4, porém agora para a análise separada dos componentes (dowels) de concreto. Para tal, manteve-se as mesmas características geométricas e de contorno dos modelos que foram descritos anteriormente na Tabela 3. Os resultados das forças máximas obtidas numericamente $\left(P_{u, \text { dowel }}\right)$ são apresentados na Tabela 4. Na última coluna dessa tabela, é efetuada a diferença entre a força máxima experimental $\left(P_{u, \text { exp }}\right)$ e a força obtida em três componentes de concreto (3 $\left.P_{u, \text { dowel }}\right)$, obtendo a parcela que é resistida pela parte frontal do conector Crestbond ( $\left.P_{u, f r o n t a l}\right)$. 
Tabela 4 - Estimativa da capacidade resistente frontal do conector Crestbond

\begin{tabular}{|c|c|c|c|c|c|c|}
\cline { 2 - 6 } \multicolumn{1}{c|}{} & $f_{\mathrm{cm}}$ & $P_{u, \exp }$ & Ds -pass & $P_{u, \text { dowel }}$ & $\begin{array}{c}3 P_{u, \text { dowel }} \\
\mathrm{kN}\end{array}$ & $\begin{array}{c}P_{u, \text { Frontal }}= \\
P_{u, \text { exp }}-3 P_{u, \text { dowel }} \\
\mathrm{kN}\end{array}$ \\
\hline Modelo & $\mathrm{MPa}$ & $\mathrm{kN}$ & $\mathrm{mm}$ & $\mathrm{kN}$ & $\mathbf{k N}$ & 177,84 \\
\hline B1-B2 & 26,60 & 301,33 & 0 & 41,16 & 123,49 & 130,62 \\
\hline B3-B4 & 27,05 & 362,30 & 10 & 77,23 & 231,68 & 142,03 \\
\hline B5-B6 & 26,65 & 374,95 & 12 & 77,64 & 232,92 & 181,43 \\
\hline C1-C2 & 47,50 & 369,40 & 0 & 62,66 & 187,97 & 194,91 \\
\hline C3-C4 & 48,90 & 500,15 & 10 & 101,75 & 305,24 & 146,17 \\
\hline C7-C8 & 49,55 & 480,90 & 12 & 111,58 & 334,73 & \\
\hline
\end{tabular}

\section{Modelo Analítico Proposto para o Cálculo do Cisalhamento do Concreto Utilizando Conectores Crestbond}

Conforme explicitado anteriormente, estudos realizados experimentalmente e numericamente por Oguejiofor e Hosain $(1994,1997)$ demonstraram que o estadolimite último que prevalece quando se utilizam conectores Perfobond é a ruptura por cisalhamento do concreto. Os autores definiram que, com esses conectores, a capacidade resistente a esse modo de colapso pode ser dada por:

$q_{u, s h}=\eta_{1} h_{s c} t_{s c} f_{c}+\eta_{2} n A_{D} \sqrt{f_{c}}+\eta_{3} A_{t r} f_{y r}$

onde $t_{s c}$ é a espessura do conector, $h_{s c}$ é a altura total do conector, $A_{D}$ é a área do componente de concreto, $A_{t r}$ é a área transversal total de armadura utilizada na laje de concreto, $n$ é o número de componentes de concreto, $f_{c}$ é a resistência do concreto à compressão e $f_{y r}$ é a resistência ao escoamento do aço da armadura.

Observando-se a Equação 3, nota-se que a capacidade resistente é definida pela soma de três parcelas. A primeira delas é devida à capacidade frontal do conector em função do fator de ajuste $\eta_{1}$, a segunda ao cisalhamento dos componentes de concreto em função do fator $\eta_{2}$, e a terceira à taxa de armadura total utilizada na laje de concreto em função do fator $\eta_{3}$. Após serem realizadas regressões analíticas, Oguejiofor e Hosain (1997) definiram esses três fatores de ajuste como 4,50, 4,21 e 0,91, respectivamente.

Em situações em que se utilizam conectores contínuos, ao se utilizar a Equação 3 é necessário: (i) desconsiderar a parcela de capacidade frontal do conector, e; (ii) substituir $A_{t r}$ por $n A_{b}$, em que $n$ é o número de componentes de concreto e $A_{b}$ a área de armadura transversal passante por componente de concreto. Dividindo-se a expressão 
pelo número $n$ de componentes de concreto, obtém-se a capacidade por componente conforme segue:

$q_{u, \text { sh, dowel }}=\eta_{2} A_{D} \sqrt{f_{c}}+\eta_{3} A_{b} f_{y r}$

Na Tabela 5 são apresentadas expressões encontradas na literatura para o cálculo da capacidade resistente por componente de concreto $\left(q_{u, s h \text {,dowel }}\right)$ de conectores contínuos formados por chapas de aço com aberturas como o Perfobond, o puzzle-shaped e o clothoidal-shaped. Por último, é apresentado o modelo analítico proposto neste trabalho para o conector Crestbond. As expressões presentes nessa tabela foram adaptadas de modo a apresentar variáveis semelhantes, facilitando a comparação entre elas. Essas adaptações estão especificadas nas observações presentes no final da tabela.

Tabela 5 - Equações para o dimensionamento de conectores contínuos constituídos por chapas com aberturas considerando o cisalhamento do concreto.

\begin{tabular}{|c|c|c|c|}
\hline Conector & Equação & Conector & Equação \\
\hline Perfobond & $\begin{array}{l}q_{u, s h}=\eta_{2} A_{D} \sqrt{f_{c}}+\eta_{3} A_{b} f_{y r} \\
\text { Fonte: Oguejiofor e Hosain (1997) }\end{array}$ & $\begin{array}{c}\text { clothoidal- } \\
\text { shaped }\end{array}$ & $\begin{array}{c}q_{u, s h}=\eta_{D} e_{x}^{2} \sqrt{f_{c}}\left[1+\rho_{d}\right]= \\
=\eta_{D} \frac{A_{D}}{0,20} \sqrt{f_{c}}\left[1+\frac{E_{r} A_{b}}{E_{c m} A_{D}}\right] \\
\text { Fonte: Z-26.4-56 (2013) }\end{array}$ \\
\hline $\begin{array}{l}\text { puzzle- } \\
\text { shaped }\end{array}$ & $\begin{array}{c}q_{u, s h}=\eta_{D} e_{x}^{2} \sqrt{f_{c}}\left[1+\rho_{d}\right]= \\
=\eta_{D} \frac{A_{D}}{0,13} \sqrt{f_{c}}\left[1+\frac{E_{r} A_{b}}{E_{c m} A_{D}}\right] \\
\text { Fonte: Z-26.4-56 (2013) }\end{array}$ & Crestbond & $\begin{array}{c}q_{u, s h}=\eta_{D} e_{x}^{2} \sqrt{f_{c}}\left[1+\rho_{d}\right] \\
=\eta_{D} \frac{A_{D}}{0,23} \sqrt{f_{c}}\left[1+\frac{E_{r} A_{b}}{E_{c m} A_{D}}\right] \\
\text { Proposto neste trabalho }\end{array}$ \\
\hline \multicolumn{4}{|c|}{$\begin{array}{l}\text { Substituições algébricas na Equação 4: } \\
\text { - } \rho_{D}=\left(E_{r} A_{b}\right) /\left(E_{c m} A_{D}\right) ; \\
\text { - para puzzle-shaped, } e_{x}^{2}=A_{D} / 0,13 \text {, e para clothoidal-shaped, } e_{x}^{2}=A_{D} / 0,20(Z-26.4-56,2013) ; \\
\text { - para Crestbond, } e_{x}^{2} \approx A_{D} / 0,23 .\end{array}$} \\
\hline
\end{tabular}

Observando-se ainda a Tabela 5, nota-se que a capacidade total de um componente de concreto ao cisalhamento utilizando conectores contínuos consiste basicamente de duas parcelas de resistência. A primeira que é função do produto da área do componente de concreto pela raiz quadrada da resistência à compressão $f_{c}$, e uma segunda que é função do acréscimo de capacidade resistente devido ao uso de armadura passante. Em Cardoso et al. (2018) verificou-se que a capacidade média por 
componente de concreto permanece constante, independentemente do número de componentes do conector.

No final do Subitem 3.3, foram propostos valores de $\eta_{D}$ para o conector Crestbond CR56b considerando o modelo analítico apresentado pela aprovação técnica Z-26.4-56 (2013). Os valores de $\eta_{D}$ que foram designados para as situações sem e com barras de armadura passante eram iguais a 0,570 e 0,873 , respectivamente.

Para utilizar o conector Crestbond CR56b descontínuo, é necessário o acréscimo da capacidade frontal do conector, fornecida pela primeira parcela da Equação 3. Para fins de praticidade, o fator $\eta_{1}$ proposto por Oguejiofor e Hosain (1994), que quantifica essa capacidade, passará a ser denominado $\eta_{F}$, de modo que a capacidade do conector Crestbond descontínuo seja dada por:

$q_{u, s h}=\eta_{F} h_{s c} t_{s c} f_{c}+n \eta_{D} e_{x}^{2} \sqrt{f_{c}}\left[1+\rho_{d}\right]$

O fator $\eta_{F}$ foi obtido a partir da estimativa de qual seria a parcela resistida pela região frontal dos conectores Crestbond CR56b ensaiados por Veríssimo (2007). Considerando que $\eta_{F}=\left(P_{u, \text { Frontal }}\right) /\left(h_{s c} t_{s c} f_{c}\right)$, e aplicando esse conceito a partir dos dados apresentados na Tabela 4, obtém-se um valor médio de $\eta_{F}$ igual a 5,059 . Esse valor é próximo do fator 4,50 estabelecido anteriormente e que define a parcela que a região frontal dos conectores Perfobond resiste. Contudo, observou-se que o valor de $\eta_{F}$ aumentava com o aumento da resistência $f_{c}$. Assim, interpolando os valores de $\eta_{F}$ em função de $f_{c}$, obtém-se:

$\eta_{F}=-0,095 f_{c}+8,310$

Com os valores de $\eta_{F}$ e $\eta_{D}$ definidos para o conector Crestbond CR56b ( $D=56 \mathrm{~mm}$, ver Figura 1a), torna-se possível o cálculo da capacidade do concreto da laje de resistir ao cisalhamento. Contudo, os valores de $\eta_{D}$ obtidos são fixados somente para a geometria de conector CR56b que apresentam valores de $e_{x}$ igual a 2,155D, ou seja, $121 \mathrm{~mm}$.

Dutra (2014) realizou um estudo numérico-paramétrico de conectores Crestbond, com o uso do software de elementos finitos Abaqus. Na série de modelos $R$, o autor variou o diâmetro $D$ do círculo inscrito no componente de concreto, sem o uso de barras de armadura passante e os conectores possuíam a mesma espessura dos modelos que foram simulados neste trabalho, com $t_{s c}$ igual a $12 \mathrm{~mm}$. De posse de seus resultados, 
foram estimados valores de $\eta_{D}$ para cada modelo dessa série utilizando as Equações 5 e 6 , e isolando-se os valores de $\eta_{D}$. Dessa forma, foi possível traçar a curva em azul apresentada na Figura 13a, que representa a variação dos valores de $e_{x}$ em relação aos valores de $\eta_{D}$. Nota-se que todos os pontos dessa função são colineares, com exceção do ponto indicado, que representa o valor $\eta_{D}$ para o conector Crestbond CR56b obtido ao final do Subitem 3.3 deste trabalho. Os demais pontos representam os resultados obtidos através dos dados disponíveis no trabalho de Dutra (2014).

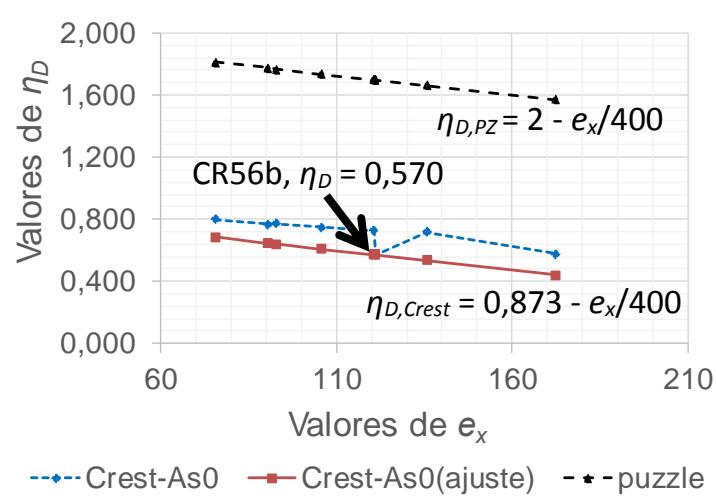

(a) Procedimento de ajuste para Crest-AsO

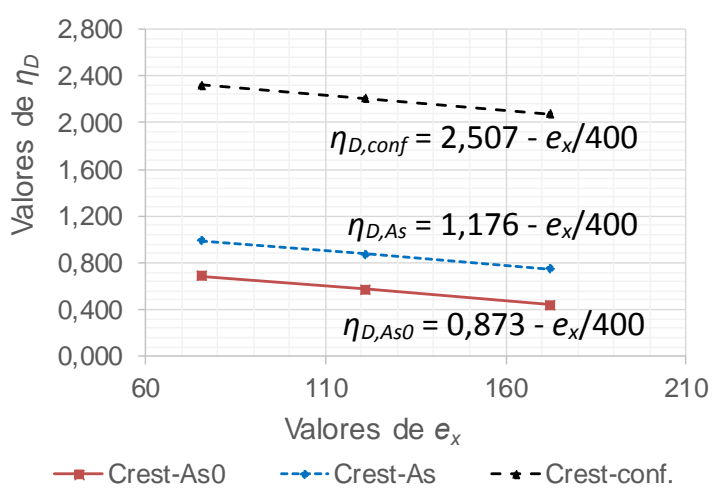

(b) $\eta_{D}$ sob condições distintas de confinamento Figura 13 - Diagrama de valores $\eta_{D}$ versus valores de $e_{x}$.

Na Figura 13a foi traçada também uma curva de cor preta representando a variação dos valores de $\eta_{D}$ em função de $e_{x}$ para o conector de geometria puzzle-shaped, com $\eta_{D, P z}=$ 2- $e_{x} / 400$ (Z-26.4-56, 2013). Essa equação é recomendada somente para situações em que o uso do conector é feito com alta taxa de armadura e alto confinamento. Apesar de que essa não seja a mesma situação a qual os conectores Crestbond estão sujeitos, onde não estão sendo utilizadas nem ao menos barras de armadura passantes (baixo confinamento), a curva do conector puzzle-shaped é útil para fins comparativos, uma vez que ela apresenta praticamente a mesma inclinação apresentada pelo conector Crestbond. Dessa forma, ajustou-se a curva deste último, de modo que ela tenha a mesma inclinação da curva do conector puzzle-shaped e que passasse pelo ponto indicado no diagrama (valor de $\eta_{D}$ igual 0,570 estimado no Subitem 3.3), obtendo-se a curva final de cor vermelha. Essa curva, portanto, caracteriza os valores de $\eta_{D}$, em função de $e_{x}$ para os conectores Crestbond quando não são utilizadas armaduras passantes.

É oportuno mencionar que o conector Crestbond pode ser utilizado também em situações com armadura passante ( $\eta_{D, A s}$ igual a 0,873, valor encontrado no Subitem 3.3), 
e com altas taxas de armadura que provocam um efeito adicional de confinamento ( $\eta_{D, \text { conf }}$ igual a 2,204, valor encontrado no Subitem 3.2). Considerando a hipótese de que o conector Crestbond mantém a mesma inclinação de curva que as apresentadas na Figura 13a, e conhecendo-se os pontos $\eta_{D, A s}$ igual a 0,873 e $\eta_{D, \text { conf }}$ igual a 2,204 , que representam a geometria de conector CR56b ( $e_{x}$ igual a $121 \mathrm{~mm}$ ), obtém-se as curvas dispostas no diagrama da Figura 13b.

Tabela 6 - Modelo analítico proposto neste trabalho para o dimensionamento de conectores Crestbond.

\begin{tabular}{|c|c|c|c|}
\hline \multicolumn{4}{|c|}{ Modelo analítico para conectores Crestbond } \\
\hline $\begin{array}{c}\text { Equação } \\
\text { geral }\end{array}$ & $\begin{array}{l}q_{u, s h}=\eta_{F} h_{s c} t_{s c} f_{c}+n \eta_{D} e \\
\text { OBS: } e_{x}=2,155 D \text { e } \rho_{D}=\left(E_{r} A_{l}\right.\end{array}$ & $\begin{array}{l}\sqrt{f_{c}}\left[1+\rho_{d}\right] \\
\left.E_{c m} A_{D}\right)\end{array}$ & \\
\hline $\begin{array}{c}\text { Valores } \\
\text { de } \eta_{F}\end{array}$ & $\eta_{F}=8,310-0,095 f_{c}$ & & \\
\hline $\begin{array}{c}\text { Valores } \\
\text { de } \eta_{D}\end{array}$ & $\begin{array}{c}\eta_{D}=0,873-e_{x} / 400 \\
\text { (nenhuma armadura passante } \\
\text { e baixo confinamento) }\end{array}$ & $\begin{array}{c}\eta_{D}=1,176-e_{x} / 400 \\
\text { (armadura passante e } \\
\text { baixo confinamento) }\end{array}$ & $\begin{array}{c}\eta_{D}=2,507-e_{x} / 400 \\
\text { (alto confinamento provocado } \\
\text { pela alta taxa de armadura) }\end{array}$ \\
\hline
\end{tabular}

Tabela 7 - Comparação entre as forças máximas dos modelos obtidos em ensaios de cisalhamento e as forças máximas estimadas através do modelo analítico.

\begin{tabular}{|c|c|c|c|c|c|c|c|c|c|c|}
\hline Modelo & $\begin{array}{c}D \\
(\mathrm{~mm})\end{array}$ & $n$ & $\begin{array}{c}e_{x} \\
(\mathrm{~mm})\end{array}$ & $\begin{array}{c}f_{c} \\
\text { (MPa) }\end{array}$ & $\rho_{D}$ & $\eta_{D}$ & $\eta F$ & $\begin{array}{c}P_{u, \text { Tеo }} \\
\mathrm{kN}\end{array}$ & $\begin{array}{c}P_{u, \mathrm{Exp}} \\
\mathrm{kN}\end{array}$ & $\frac{P_{u, T e o}}{P_{u, \text { Exp }}}$ \\
\hline B1* & 56 & 3 & 121 & 26,60 & 0,00 & 0,570 & 5,783 & 279,32 & 296,20 & 0,943 \\
\hline B2* & 56 & 3 & 121 & 26,60 & 0,00 & 0,570 & 5,783 & 279,32 & 306,50 & 0,911 \\
\hline B3* & 56 & 3 & 121 & 27,20 & 0,16 & 0,873 & 5,726 & 384,88 & 348,80 & 1,103 \\
\hline B4* & 56 & 3 & 121 & 26,90 & 0,16 & 0,873 & 5,755 & 382,78 & 375,90 & 1,018 \\
\hline B5* & 56 & 3 & 121 & 28,50 & 0,23 & 0,873 & 5,603 & 408,27 & 378,20 & 1,080 \\
\hline B6* & 56 & 3 & 121 & 24,80 & 0,24 & 0,873 & 5,954 & 381,48 & 371,80 & 1,026 \\
\hline C1* & 56 & 3 & 121 & 46,90 & 0,00 & 0,570 & 3,855 & 348,00 & 361,30 & 0,963 \\
\hline $\mathrm{C} 2^{*}$ & 56 & 3 & 121 & 48,10 & 0,00 & 0,570 & 3,741 & 349,35 & 377,50 & 0,925 \\
\hline C3* & 56 & 3 & 121 & 49,10 & 0,14 & 0,873 & 3,646 & 480,49 & 493,70 & 0,973 \\
\hline C4* & 56 & 3 & 121 & 48,70 & 0,14 & 0,873 & 3,684 & 479,72 & 506,70 & 0,947 \\
\hline C5* & 56 & 3 & 121 & 48,70 & 0,20 & 0,873 & 3,684 & 495,88 & 465,00 & 1,066 \\
\hline C6* & 56 & 3 & 121 & 45,90 & 0,20 & 0,873 & 3,950 & 489,28 & 496,90 & 0,985 \\
\hline R35** & 35 & 5 & 75 & 25,00 & 0,00 & 0,684 & 5,935 & 204,19 & 221,00 & 0,924 \\
\hline $\mathrm{R} 42^{* *}$ & 42 & 3 & 91 & 25,00 & 0,00 & 0,647 & 5,935 & 198,77 & 214,00 & 0,929 \\
\hline $\mathrm{R} 43^{* *}$ & 43 & 4 & 93 & 25,00 & 0,00 & 0,641 & 5,935 & 231,23 & 254,00 & 0,910 \\
\hline R49** & 49 & 3 & 106 & 25,00 & 0,00 & 0,609 & 5,935 & 233,63 & 257,00 & 0,909 \\
\hline R56** & 56 & 3 & 121 & 25,00 & 0,00 & 0,571 & 5,935 & 269,04 & 303,00 & 0,888 \\
\hline R63** & 63 & 3 & 136 & 25,00 & 0,00 & 0,534 & 5,935 & 304,23 & 356,00 & 0,855 \\
\hline R80** & 80 & 2 & 172 & 25,00 & 0,00 & 0,442 & 5,935 & 318,33 & 358,00 & 0,889 \\
\hline \multicolumn{11}{|c|}{$\begin{array}{l}\text { *Modelo experimental analisado por Veríssimo (2007); }{ }^{* *} \text { Modelo numérico analisado por Dutr } \\
\text { (2014). } \\
\text { - Todos os modelos apresentavam as seguintes caraterísticas geométricas: } \\
\qquad t_{s c}=12,0 \mathrm{~mm} \text { e } h_{s c}=D+c_{u} ; \text { onde } c_{u}=25 \mathrm{~mm} \text {, é o cobrimento inferior (Figura 1a) }\end{array}$} \\
\hline
\end{tabular}


Considerando todos os passos realizados neste trabalho, na Tabela 6 é apresentado um quadro resumo contendo o modelo analítico para obtenção da capacidade resistente de conectores Crestbond, em que a ruptura por cisalhamento do concreto governa o colapso da conexão. Na Tabela 7 são apresentadas as forças máximas dos modelos de ensaios de cisalhamento com conectores Crestbond $\left(P_{u, E x p}\right)$, encontradas na literatura, e as forças teóricas $\left(P_{u, T e o}\right)$, estimadas através do modelo analítico proposto neste trabalho (Tabela 6). Na última coluna é realizada a razão entre as forças $P_{u, T e o} / P_{u, \text { Exp }}$, obtendo uma razão média de 0,96.

\section{Conclusões}

Este artigo apresenta um modelo analítico para o dimensionamento de conectores Crestbond quando o estado-limite último é o cisalhamento da laje de concreto. Esse modelo segue a tendência de padronizar as expressões para o dimensionamento dos conectores de geometria aberta, conhecidos como composite dowels. Ao final, o modelo analítico foi comparado com resultados encontrados na literatura e aos que foram obtidos nas simulações deste trabalho, obtendo-se uma boa concordância entre esses resultados.

Na expressão utilizada para obter a capacidade ao cisalhamento dos componentes de concreto, é utilizado o fator $\eta_{D}$ que depende da geometria do conector. Caso o conector seja utilizado de forma intermitente, deve ser acrescida a parcela referente à resistência frontal do conector. A capacidade frontal do conector é função do fator $\eta_{F}$ que varia linearmente com a resistência do concreto. Neste trabalho foram desenvolvidas expressões para determinar os valores de $\eta_{D}$ e $\eta_{F}$ para qualquer geometria de conector Crestbond.

Caso se deseje utilizar o conector Crestbond contínuo, recomenda-se utilizar barras de armadura passantes nos componentes de concreto. Essas barras contribuem para uma melhor ductilidade da conexão, evitando fissuras prematuras que podem afetar a capacidade do conector e a durabilidade da laje de concreto.

\section{Agradecimentos}

Os autores agradecem o aporte financeiro concedido pela CAPES (Coordenação de Aperfeiçoamento de Pessoal de Nível Superior), CNPq (Conselho Nacional de 
Desenvolvimento Científico e Tecnológico) e FAPEMIG (Fundação de Amparo à Pesquisa

do Estado de Minas Gerais).

\section{Referências bibliográficas}

Allgemeine bauaufsichtliche Zulassung der Verbunddübelleiste, No. Z-26.4-56, Deutsches Institut für Bautechnik, Berlin, 2013.

Cardoso, S. H.; Caldas, R. B.; Fakury, R. H. Estudo do Comportamento de Conectores Crestbond por meio de Simulação Numérica. Revista da Estrutura de Aço - REA, v.xx(X), p. xxx-xxx, 2018.

Classen, M.; Gallwoszus, J. Concrete Fatigue in Composite Dowels. Structural Concrete, v.17(1), p. $63-73,2016$.

Classen, M.; Hegger, J. Assessing the Pry-out Resistance of Open Rib Shear Connectors in Cracked Concrete - Engineering Model with Aggregate Interlock. Engineering Structures, v.148, p. $254-262,2017$.

Dutra, C. M. Estudo do Comportamento Estrutural do Conector Crestbond Considerando Variações Geométricas e Mecânicas. Dissertação de Mestrado - Universidade Federal de Viçosa (UFV), Viçosa, Brasil, 2014.

Feldmann, M.; Kopp, M.; Park, D. Composite Dowels as Shear Connectors for Composite Beams - Background to the German Technical Approval. Steel Construction, v.9(2), p. 80-88, 2016.

Hibitt, Karlsson, Sorensen. Abaqus Online Documentation. Version 6.14, Dassault Systèmes, SIMULIA, 2014.

Oguejiofor, E. C.; Hosain, M. U. A parametric Study of Perfobond Rib Shear Connectors. Canadian Journal of Civil Engineering, v.21(4), p. 614-625, 1994.

Oguejiofor, E. C.; Hosain, M. U. Numerical Analysis of Push-out Specimens with Perfobond Rib Connectors. Computers \& Structures, v.62(4), p. 617-624, 1997.

Přivřelová, V. (2016). Verification and Actual Behavior of Shear Connection of Composite Steel and Concrete Structures Using Pcb-W Wechnology. International Journal of Mechanics, v.10, p. 356-361, 2016.

Seidl, G.; Viefhues, E.; Berthellemy, J.; Mangerig, I.; Wagner, R.; Lorenc, W.; Kozuch, M.; Franssen, J-M.; Janssen, D; Ikäheimonen, J.; Lundmark, R.; Hechler, O; Popa, N. Preco-Beam: Prefabricated Enduring Composite Beams Based on Innovative Shear Transmission. Research Fund for Coal and Steel - Final Report. Luxemburgo, Luxemburgo, 2013a.

Seidl, G.; Hoyer, O.; Zanon, R.; Popa, N.; Lorenc, W, Rowiński, S.; Kożuch, M.; Franssen, J-F., Fohn, T.; Quinchia, J. P.; Carrasco, C. H.; Farhang, A.; Nüsse, G. PRECO+: Prefabricated Enduring Composite Beams Based on Innovative Shear Transmission. Research Fund for Coal and Steel - Final Report. Berlin, Alemanha, 2013b.

Silva, M.A.P. Modelos Semiempíricos para a Estimativa da Capacidade Resistente de Conectores de Cisalhamento Crestbond e Perfobond. Dissertação de Mestrado - Universidade Federal de Viçosa (UFV), Viçosa, Brasil, 2011.

Veríssimo, G. S. Desenvolvimento de um Conector de Cisalhamento em Chapa Dentada para Estruturas Mistas de Aço e Concreto e Estudo do seu Comportamento. Tese de Doutorado Escola de Engenharia, Universidade Federal de Minas Gerais (UFMG), Belo Horizonte, Brasil, 2007. 